9 Falagas ME, Vardakas KZ, Samonis G. Decreasing the incidence and impact of infections in neutropenic patients: evidence from meta-analyses of randomized controlled trials. Curr Med Res Opin 2008; 24: 215-235.
10 Singh A. Fluoroquinolones should not be the first-line antibiotics to treat community-acquired pneumonia in areas of tuberculosis endemicity. Clin Infect Dis 2007; 45: 133.

\title{
Nebulised corticosteroid and amphotericin B: an alternative treatment for ABPA?
}

\section{To the Editors:}

Allergic bronchopulmonary aspergillosis (ABPA) is a pulmonary disease that results from a hypersensitivity response to Aspergillus fumigatus [1]. It occurs in asthmatic and cystic fibrosis (CF) patients, with a prevalence among CF patients recently estimated at $7.8 \%$ [1]. The natural course is characterised by acute exacerbations, which can be life-threatening [2], and progressive, irreversible, lung damage leading to longterm pulmonary fibrosis if the disease is not well controlled [1].

Therapy for ABPA has two main targets. The first is the attenuation of the inflammation and immunological activity, for which oral corticosteroids are the mainstay of therapy [1]. The second is the attenuation of the antigen burden arising from fungal colonisation of the bronchial tree, for which only itraconazole has shown limited evidence of its usefulness [1]. However, long-term systemic corticotherapy predisposes to diabetes, osteopaenia, infections and growth retardation, especially in patients with $\mathrm{CF}$, while itraconazole is associated with several drug interactions, suppression of adrenal glucocorticoids synthesis when associated with inhaled budenoside [3], and hepatic toxicity [4,5]. After one of our patients experienced serious side-effects while treated for ABPA with oral corticosteroids, it was decided that a different drug regimen should be used for this disease. We report herein three cases of CF patients with ABPA successfully treated by the association of nebulised budesonide (Nbud) and nebulised amphotericin B (NampB): three children of 7, 12 and 13 yrs of age affected by $C F$ with $\Delta 508$ mutation, two of whom were chronically infected with Staphylococcus aureus and one with Pseudomonas aeruginosa. The oldest child had developed diabetes.

The three cases benefited from a treatment that associated chest physiotherapy, aerolised recombinant DNase, inhaled aminoglycosides and regular outpatient intravenous antibiotic therapy through a central catheter. In a context of well-controlled disease, these patients developed wheezing dyspnoea, chest pain, new infiltrates on chest radiography and decreased pulmonary function. Additional studies demonstrated peripheral blood eosinophilia, an increase in total serum immunoglobulin (Ig)E and specific IgE, and precipitins to A. fumigatus. The diagnosis of ABPA was established according to the Cystic Fibrosis Foundation Consensus Conference [1].

Cases 1 and 2 benefited from two successive treatments. First, they received an association of Nbud ( $1 \mathrm{mg}$ twice a day) and oral itraconazole (400 mg daily) for almost $1 \mathrm{yr}$. Their clinical status improved but due to the lack of improvement of proximal obstruction, hypoxaemia and the persistence of high levels of precipitatins and serum IgE antibodies to A. fumigatus, itraconazole was changed to $\mathrm{NamB}$ ( $5 \mathrm{mg}$ twice a day), while maintaining Nbud at the same dose. Due to a dramatic response in serological status for both cases, case 3 benefited directly from the association of Nbud and NampB. The three children presented a good clinical, biological and functional response to this treatment, from the 6th month on, with improvement of hypoxaemia and forced expiratory volume in one second, net reduction of blood eosinophilia, total and specific serum IgE antibodies to A. fumigatus and a disappearance of precipitatins to A. fumigatus.

To our knowledge, the three cases reported herein are the first cases of ABPA to have benefited from the association of Nbud and NampB. Previous articles have shown the usefulness of inhaled corticosteroids in ABPA but always as a means of discontinuing an oral corticotherapy [6]. Another study has failed to support these findings; however, the dose of inhaled corticosteroids was very low [7]. A recent article reviewed antifungal therapies in the treatment of ABPA, and NampB showed good results for two patients but always in association with oral prednisone [4]. However, our findings support the fact that ABPA can be treated without systemic corticosteroids in patients with CF. The association of Nbud and NampB resulted in a complete clinical remission of $\mathrm{ABPA}$ and a substantial improvement of all biological and functional parameters. Cases 1 and 2 first received Nbud associated with itraconazole and showed a good but incomplete response. Then the switch from itraconazole to NampB allowed a complete cure of ABPA. Case 3 benefited straight away from the association of Nbud and NampB, and showed a quick and complete cure. Moreover, none of the cases have shown any side-effects.

This new treatment is two-fold: Nbud decreases the inflammation and immunological activity, while NampB decreases the antigenic load of $A$. fumigatus. The use of nebulised drugs greatly limited the toxicity of the treatment.

In conclusion, we think the association of nebulised budenoside and nebulised amphotericin B is a safe and efficacious treatment of allergic bronchopulmonary aspergillosis in patients with cystic fibrosis. We also feel that this nebulised treatment could greatly benefit asthmatic patients with allergic bronchopulmonary aspergillosis, as its long-term side-effects 
are much lower than those of oral corticotherapy. We recommend that large, controlled studies compare this treatment to oral corticosteroids in cases of allergic bronchopulmonary aspergillosis, both in cystic fibrosis and asthmatic patients.

\section{Y. Laoudi, J-B. Paolini, A. Grimfed and J. Just}

Dept of Multidisciplinary Paediatrics, Centre of Asthma and Allergies of the Child, Public Assistance of Paris' Hospitals, Hospital Group Trousseau/La Roche Guyon, Pierre and Marie Curie Univeristy, Paris, France.

\section{STATEMENT OF INTEREST}

None declared.

\section{REFERENCES}

1 Stevens DA, Moss RB, Kurup VP, et al. Allergic bronchopulmonary aspergillosis in cystic fibrosis - state of the art: Cystic Fibrosis Foundation Consensus Conference. Clin Infect Dis 2003; 37: Suppl. 3, S225-S264.
2 Skowronski E, Fitzgerald DA. Life-threatening allergic bronchopulmonary aspergillosis in a well child with cystic fibrosis. Med J Aust 2005; 182: 482-483.

3 Skov M, Main KM, Sillesen IB, Muller J, Koch C, Lanng S. Iatrogenic adrenal insufficiency as a side-effect of combined treatment of itraconazole and budesonide. Eur Respir J 2002; 20: 127-133.

4 Leon EE, Craig TJ. Antifungals in the treatment of allergic bronchopulmonary aspergillosis. Ann All Ast Immunol 1999; 82: 511-517.

5 Elphick H, Southern K. Antifungal therapies for allergic bronchopulmonary aspergillosis in people with cystic fibrosis. Cochrane Database Syst Rev 2000; 4: CD002204.

6 Seaton A, Seaton RA, Wightman AJ. Management of allergic bronchopulmonary aspergillosis without maintenance oral corticosteroids: a fifteen-year follow-up. QJM 1994; 87: 529-537.

7 British Thoracic Association, Inhaled beclomethasone diproprionate in allergic bronchopulmonary aspergillosis. $\mathrm{Br} \mathrm{J} \mathrm{Dis}$ Chest 1979; 73: 349-356.

\section{Elevated levels of exhaled nitric oxide in recurrent tonsillitis}

\section{To the Editors:}

Nitric oxide (NO) is a free radical generated from L-arginine through the NO synthases (NOS). This molecule is produced within the respiratory tract and plays an important role in airway pathophysiology, exerting both beneficial and nonbeneficial effects $[1,2]$.

Because various pro-inflammatory mediators and lipopolysaccharides are capable of inciting inducible NOS (iNOS) expression [2], different airway inflammatory diseases are associated with increased NO release, which is detectable in exhaled gas, including asthma [3], bronchiectasis [4], allergic rhinitis [5] and upper respiratory tract infections [6]. However, not all sources of the elevated exhaled NO (eNO) have been identified as yet. Interestingly, it has been suggested that iNOS expression and formation of peroxynitrite may play an important role in the pathogenesis of recurrent tonsillitis [7]. To investigate whether recurrent tonsillitis is associated with increased levels of eNO, we measured oral eNO levels in adult patients with a history of frequent tonsillitis episodes.

The study group consisted of 32 nonatopic, nonsmoking patients with recurrent tonsillitis, scheduled for tonsillectomy (18 females; median (range) age $28(18-50)$ yrs). All patients had a history of frequent episodes of tonsillitis over many years. All patients had received antibiotic treatment $>1$ month prior to surgery. Specific exclusion criteria were diseases that may affect eNO concentration, including asthma, rhinitis and sinusitis. Routine histological examination of tonsillectomy was performed for each of the cases.

A total of 35 nonatopic, nonsmoking healthy subjects (20 females; age 30 (18-49) yrs) were included in the control group. Oral eNO levels were measured in triplicate using the Niox Mino ${ }^{\circledR}$ analyser (Aerocrine AB, Solna, Sweden). The level of NO is expressed in ppb.

Statistical analysis was performed using the Mann-Whitney U-test. A p-value $<0.05$ was considered significant.

eNO levels were significantly higher in patients with recurrent tonsillitis $(21.0(6.0-50.0) \mathrm{ppb})$ than in normal controls (17.0 (5.0-26.0) ppb; $\mathrm{p}<0.05$; fig. 1). This suggests that recurrent tonsillitis may increase the level of NO in exhaled air. eNO can be a sensitive marker of inflammation in the respiratory tract but is not specific for asthma, as increased levels of oral eNO have been observed in other diseases, including infections of the upper respiratory tract [6]. However, NO levels did not differ between healthy subjects and common cold patients, and were significantly lower in patients suffering from chronic sinusitis [8] as well as from acute sinusitis [9].

The present study was the first to show that, to the best of our knowledge, oral exhaled nitric oxide can be elevated in patients with recurrent tonsillitis, probably as a result of the 